\title{
A NEED OF BETTER PHARMACOVIGILANCE SYSTEM IN INDIA
}

\author{
PRAJAKTA MASURKAR*
}

Department of Drug Development and Regulatory Sciences, SVKM'S, Shobhaben Pratapbhai Patel School of Pharmacy and Technology Management, NMIMS, Mumbai, Maharashtra, India. Email: prajaktapmasurkar@gmail.com

India is one of the top producers of generics globally and is currently being recognized as the "pharmacy of the world" for the generic drug products. Thus, there is a need that it has best health-care systems in place to regulate and provide better quality drugs by monitoring the possible risk associated with the use of drugs. Being the generic hub, physicians, pharmacists, etc., should be erudite to provide an alternative cost-effective generic medicine, which is one of the education-related aspects of pharmacovigilance. We need a more systematic approach to surveillance of drug-related problems, which is at the heart of pharmacovigilance. The health-care system requires new processes to understand the risk-benefit ratio of drugs. The challenges in implementation of better pharmacovigilance in country due to nonavailability of trained staff in pharmacovigilance, lack of training of health-care professionals on drug safety, and adverse drug reaction reporting which comprises adverse interactions of medicines with chemicals, other medicines, and food are often neglected leading to under-reporting by health-care professionals as well as patients, lack of expertise, etc., should be overcome by Indian regulatory body via practical oriented knowledge-based system. The web market monitoring, global electronic database, education, association of stakeholders and regulation of herbal medicines standards and allied medicinal systems are vital restructurings needed to be introduced for a better pharmacovigilance system in India.

Keywords: Pharmacovigilance, India.

(C) 2017 The Authors. Published by Innovare Academic Sciences Pvt Ltd. This is an open access article under the CC BY license (http://creativecommons. org/licenses/by/4. 0/) DOI: http://dx.doi.org/10.22159/ajpcr.2017.v10i1.14797

\section{INTRODUCTION}

As per the World Health Organization (WHO), pharmacovigilance is the "Science and activities relating to the detection, assessment, understanding and prevention of adverse effects (AEs) or any other drug-related problem" [1]. Every drug has its beneficial pharmacological activity as well unavoidable side effects. The risk can be minimized by better quality drugs, awareness among patients and the right therapeutic decision by health-care professionals. Pharmacovigilance helps in reducing these risks by understanding the safety profile of drugs and forecasts the serious adverse drug reaction (ADR) based on the information. Pharmacovigilance has not developed in India to that extent than it has in western countries. Reasons: Inadequacy of the health-care system, lack of awareness among people regarding proper usage of medicines and no urgency by the government to establish the proper pharmacovigilance system. In the USA, the laws regarding drug safety monitoring were applied in 1962, in Europe around 1963-1964. However, in India 1997, this when India joined WHO-ADR monitoring program with 3 centers nationwide [2]. However, an appreciable program has been initiated by Central Drugs Standard Control Organization (CDSCO) under Government of India, a nationwide pharmacovigilance program in July 2010, for monitoring ADR in the country to safe-guard public health [3].

In recent years, India has become hub for clinical research activities due to its large population, high enrolment rate, and low cost. However, its first time availability in the USA or European market and later in India, results in unavailability of long-term safety data of such drugs. In such scenarios, Indian regulatory bodies cannot rely on safety data of other markets to assess the risk-benefit balance of a drug, thereby implying the importance of our very own pharmacovigilance systems.

\section{PHARMACEUTICAL COMPANIES}

Pharmaceutical companies are one of the keys connect in the chain of pharmacovigilance system. The Indian regulatory agency should make pharmacovigilance reporting mandatory for all pharmaceutical companies who are marketing their drugs in India. Pharma companies should maintain database all ADRs reported to them via consumers or health-care professionals. A pharmaceutical company can accomplish this either by setting up in-house systems for pharmacovigilance or by contractual agreements with clinical research organizations (CROs) [4]. The company should be accountable to submit ADRs postmarketing of drugs and even during the clinical trials. The regulatory agency should introduce pharmacovigilance inspections. The companies should have ethical consideration for event reporting during clinical trials as well as postmarketing of the drugs. To reduce the administrative burden, they can outsource work to different CROs. These practices are already followed by global companies who have affiliates across the world nevertheless; Schedule Y of the Drugs and Cosmetics Act 1945 does not insist on anything concerning the expedited reporting of serious adverse reactions from other nations. This is one of the major loopholes in pharmacovigilance structure of India [5].

\section{DRUG USAGE}

Most of the ADRs reported worldwide are related to improper drug administration and incorrect usage. Examples of such ADRs are medication errors, lack of efficacy reports, intentional drug misuse, drug abuse, improper administration of the drug especially injectables, injection site reactions [6]. Public awareness should be created regarding the drug use. Reducing ADR occurrences can be a highlight for effective pharmacovigilance. The drug usage should also be taken into account and safety profile of the drug on its utilization pattern can be monitored. The drug utilization studies should be carried out based on the pattern of use, quality of use, and outcome of use. This will give an idea for ADR occurrences and can be relevant for drug development and prescription practices. It also provides insight into the efficiency of drug use, i.e., whether a certain drug therapy provides value for money. The benefit, harm, effectiveness, and risk of medicines should be communicated to the public and patients to circumvent ADRs as well 
as to make them aware consumption of drugs. For instance, during a pandemic of H1N1 influenza, European Medicines Agency issued a press release on reiterating the efficacy and safety of the centrally authorized vaccines, it turned out to be affirmative, and no unforeseen serious safety issues had been identified [7]. There is need of rational drug usage. ADRs cases can be associated with the patterns of drug use within the public, which arises due to unreasonable drug use, drug interactions, medication errors, self-medication, etc.

\section{GLOBAL ELECTRONIC DATABASE}

With the help of IT sector, vigorous electronic database for pharmacovigilance system of India should be built. It should provide risk-benefit ratio of the drug via computer algorithms that statistically measures the reporting frequency for huge numbers of drug-event combinations are increasingly and helps screening large spontaneous reporting system databases and review of health statistics and drug utilization data [8]. The databases containing patient profiles, physician profiles, clinical trials ADRs, spontaneous reporting submissions, etc., should be established. The database mustincorporate Medical Dictionary for Regulatory Activities and E2B to follow international guidelines. The database will help to identify specific product $\mathrm{AE}$ combinations against marginal distributions as a background and can be used for research purposes too. It should also include drug information and their ADRs listings as per the individual pharmaceutical company. A data-driven framework for managing the pharmacovigilance of marketed products is needed to be launched. The database must, therefore, be validated and acceptable to regulatory authorities. The WHO Collaborating Centre, the Uppsala Monitoring Centre, can act as guidance for the required purpose, which is responsible for the management of the WHO program for international drug monitoring [9]. Data quality is crucial to institute causality which is the most vital characteristic in the investigation of suspect ADR reports. This aids in founding the probable relationship of the reaction with the drug. If the reaction is conclusively due to the drug supplementary safety measures can be taken while prescribing the same medication [10]. Even the statistical method can be used to execute organized signal detection of adverse event information.

\section{EDUCATION}

It means both existing health-care professionals as well as infant stage; students of the health-care system and layman should be educated about the importance of pharmacovigilance. Physicians, pharmacists, and nurses play a chief role in pharmacovigilance as they are first to detect AEs in patients admitted for treatment [11]. They can educate patients and their families for the signs and symptoms that should be reported immediately. Hence, they must have accurate and appropriate knowledge about the same for better $\mathrm{AE}$ reporting. Thus, encourage compliance with the ADR reporting program. The awareness programs, training, and usage of digital media to reach to public for the safeguard of their health must be used to educate people regarding drugs and potential ADRs. Adverse reactions tend to be regarded, falsely, as "side effects" which would lead to wrong signaling of the drug safety report, to avoid all these health professionals and regulators should be well educated and trained about good safety monitoring. The pharmacy educational institutions must integrate pharmacovigilance courses in the curriculum as one of the subjects to give first-hand experience of the same [12]. Incessant education of health-care personnel about pharmacovigilance by oral demonstrations, verbal aides-mémoires, delivering ADR newsletters/bulletin/case reports by email, mailing and direct supply for hospital staff, billboard, presence of pharmacist in the medical wards and involving enthusiastically in education and training of health-care staffs, especially nurses and physicians are needed for development of knowledge, alertness and outlook of health-care personnel about ADRs [45].

\section{INDIAN REGULATORY AGENCY}

The most important and chief link in pharmacovigilance system. The Pharmacovigilance Programme of India initiated in 2010 is a step forward by CDSCO and Ministry of Health and Family Welfare,
Government of India [14]. It should develop a uniform structure for reporting of events nationwide including creating a single nationwide specific adverse event reporting form to be used by all, making core teams of trained scientific and medical expertise, having global knowledge for developing the efficient health-care system.

There is in need to set up our own pharmacovigilance monitoring center of global standards. It includes having a national database or system for collating and managing ADR reports, the existence of a national spontaneous reporting system with a national ADR reporting form, training of staff in case assessment, signal analysis and benefitrisk evaluation, access to the VigiFlow software for management of individual case safety reports [15]. Furthermore, it requires collaborating with pharmacovigilance organizations in enhancing drug safety, working together with CROs, hospitals to train patient as well as health-care professional for effective adverse event reporting. National Monitoring Centers are important in increasing public awareness of drug safety and communication of this information to patients and practitioners.

\section{WEB MARKET MONITORING}

In this digital age, increasing use of the Internet has resulted in a change in access to all pharmaceutical products and information on them, giving rise to need of more vigilant safety measures. The introduction of e-commerce in the world, which provided convenience to consumers along with offers, discounts, and home-delivered products over the past decade, also led to the development of the cyber pharma drug market [16]. The last decade has seen the rise of new internet tools that have acted as important enablers of online drug markets. The Internet now hosts a range of virtual marketplaces for selling and buying illicit substances, as well as signifying a new arena for health and law enforcement involvements [17]. The practices such as illegal sale of medicines without prescriptions over the Internet, growing selfmedication practices, manufacture, and sale of counterfeit low-quality medicines should be detected to be controlled and curtailed. Therefore, in India, the need for regulating online drug sales is as pivotal to health care as is the need to have drug sale and distribution regulated, otherwise. We need to develop active relationships with the media to facilitate effective and accurate communication of drug information to the public.

\section{ASSOCIATION OF STAKEHOLDERS}

The pharmacists, clinicians, pharmaceutical manufacturers, drug developers, regulators, public lawmakers, patients, and the general public all have their own harmonizing roles in achieving what is envisioned [18]. Patients should also be helped to understand that the information they provide is likely to contribute to an international understanding of drug safety. There is need to develop a platform that encourages debate and discussion between regulators, manufacturers, consumers, and the media that would ensure much toward achieving effective pharmacovigilance objectives. The strengthening medicine safety transparency and communication so that the understanding and trust of patients and health professionals in the safety of medicines will improve, as well as the penetration of key cautions will lead to the better long-term running system [19].

\section{REGULATION OF HERBAL MEDICINES AND ALLIED MEDICINAL SYSTEMS}

Herbal medicines or ayurvedic medicines are widely used all over the country. However, the reports regarding ADR reporting with them is low. This does not give an indication anything herbal is safe. It is one of the drawbacks that adverse reaction terminology for herbal or ayurvedic drugs is not well versed. An even drug-drug interaction is overlooked while reporting. The National Pharmacovigilance Centre for Ayurveda, Siddha, and Unani (ASU) drugs in India was established taking the WHO guidelines for the safety issues of herbal medicines into consideration [19]. However, awareness among the public regarding the same needs to be focused. A standardization of herbal medicines is 


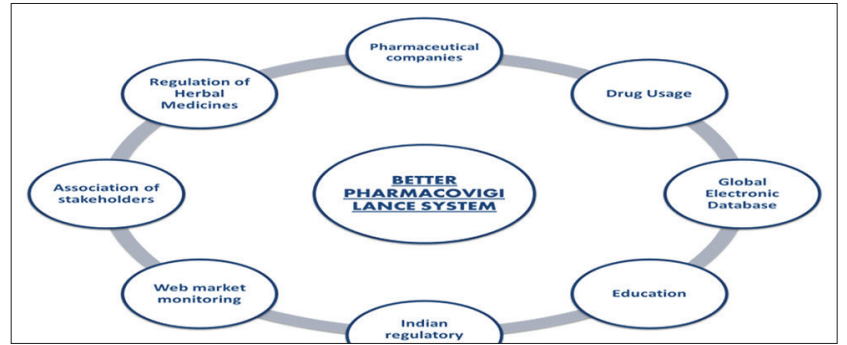

Fig. 1: Factors to be considered for better pharmacovigilance in India

required for getting accepted in the global market as well as identifying the adverse reaction caused by them. The herbal medicines behave pharmacologically different than allopathic medicines, and thus, ADRs manifested are different based on pharmaceutics of a drug. On the lines of WHO database for herbs and drug interactions, there is a need to develop safety database for ASU system of medicines [20]. The monitoring the safety of herbal medicinal products will definitely go a long way in restoring the confidence of their safety.

\section{CONCLUSION}

The Drug Controller General of India has shown its commitment to ensure the safe use of drugs by establishing the National Pharmacovigilance Program. The challenges in the implementation of better pharmacovigilance in the country due to nonavailability of trained staff in pharmacovigilance, lack of training of health-care professionals in drug safety and ADR reporting, lack of expertise, etc., should overcome by Indian regulatory body via knowledge-based systems. For an effective pharmacovigilance system to be functional and efficient, all the stakeholders need to be alert and attentive throughout the lifecycle of a medicinal product in the market. The health-care professionals, patients, and pharmaceutical companies should report ADRs by own selves and actively participate in the pharmacovigilance system of the country. The system needs to be reviewed timely to face future challenges. Ultimately, an effective pharmacovigilance will facilitate the competent pool of data from all sources, transformation of significant data into information with authorization of the country to use this information, enhancing the trends of medicine use in their countries, and enable to make judicious therapeutic judgments on the usage of medicine. The progression of pharmacovigilance cannot occur in inaccessibility; rather, it must be part of a larger effort to improve global clinical research and development and reform the regulatory system.

\section{REFERENCES}

1. WHO. Definition of Pharmacovigilance. Geneva: WHO; 2002.

2. Ahmad A, Patel I, Sanyal S, Balkrishnan R, Mohanta GP. A study on drug safety monitoring program in India. Indian J Pharm Sci 2014;76(5):379-86.

3. CDSCO, Ministry of Health and Family Welfare, Government of India. Pharmacovigilance Programme of India. New Delhi: CDSCO, Ministry of Health and Family Welfare, Government of India; 2010.

4. Almenoff JS. Innovations for the future of pharmacovigilance. Drug Saf 2007;30(7):631-3.

5. Arora D. Pharmacovigilance obligations of the pharmaceutical companies in India. Indian J Pharmacol 2008;40 Suppl 1:S13-6.

6. Brahmachari B. Pharmacovigilance for clinical trials in India: Current practice and areas for reform. Perspect Clin Res 2011;2(2):49-53.

7. Pandemic Pharmacovigilance Weekly Update Status, 2009. Available from: http://www.ema.europa.eu/pdfs/influenza/78468109en.pdf. [Last cited on 2010 Jan 12].

8. Bates DW, Gawande AA. Improving safety with information technology. N Engl J Med 2003;348(25):2526-34

9. CIOMS Working Group IV. Benefit-risk Balance for Marketed Products: Evaluating Safety Signals. Geneva: Council for International Organizations of Medical Sciences (CIOMS); 1999.

10. Roy K, Divya S, Nadig P, Prakash B. Monitoring and analysis of adverse drug reactions in a private tertiary care teaching hospital. Asian J Pharm Clin Res 2015;8(2):337.

11. Eisenhauer LA. Adverse drug reactions: A concern for clinicians and patients. Clin Excell Nurse Pract 2002;6:3-7.

12. El Emam K, Jonker E, Sampson M, Krleza-Jeric K, Neisa A. The use of electronic data capture tools in clinical trials: Web-survey of 259 Canadian trials. J Med Internet Res 2009;11(1):e8.

13. European Monitoring Centre for Drugs and Drug Addiction. The Internet and Drug Markets. Lisbon: European Monitoring Centre for Drugs and Drug Addiction; 2016.

14. Barratt MJ, Lenton S, Allen M. Internet content regulation, public drug websites and the growth in hidden internet services. Drugs Educ Prev Policy 2013;20(3):195-202.

15. Edwards B, Olsen AK, Whalen MD, Gold MJ. Guiding principles of safety as a basis for developing a pharmaceutical safety culture. Curr Drug Saf 2007;2(2):135-9.

16. Borg JJ, Aislaitner G, Pirozynski M, Mifsud S. Strengthening and rationalizing pharmacovigilance in the EU: Where is Europe heading to? A review of the new EU legislation on pharmacovigilance. Drug Saf 2011;34(3):187-97.

17. Baghel M. The national pharmacovigilance program for Ayurveda, Siddha and Unani drugs: Current status. Int J Ayurveda Res 2010;1(4):197-8

18. Chaudhary A, Singh N, Kumar N. Pharmacovigilance: Boon for the safety and efficacy of Ayuvedic formulations. J Ayurveda Integr Med 2010;1(4):251-6.

19. Report of CIOMS (Council for International Organisations of Medical Sciences) Working Group III. Guidelines for Preparing Core ClinicalSafety Information on Drugs. Geneva: Council for International Organisations of Medical Sciences; 1995

20. CIOMS Working Group VIII. Practical Aspects of Signal Detection in Pharmacovigilance. Geneva: Council for International Organizations of Medical Sciences (CIOMS); 2010

21. International Conference on Harmonisation of Technical Requirements for Registration of Pharmaceuticals for Human Use (ICH). Clinical Safety Data: Data Elements for the Transmission of Individual Case Safety Reports E2B (R2). Step 4 Dated 05 February, 2001.

22. International Conference on Harmonisation of Technical Requirements for Registration of Pharmaceuticals for Human Use (ICH). Guideline for Good Clinical Practice E6 R1. Step 4 Dated 10 June, 1996. Available from: http://www.ich.org/fileadmin/Public_Web_Site/ICH_Products/ Guidelines/Efficacy/E6/E6 R1 Guideline.pdf. [Last accessed on 2016 Jan 06].

23. US Department of Health and Human Services Food and Drug Administration, Center for Drug Evaluation and Research (CDER), Center for Biologics Evaluation and Research (CBER). Safety Assessment for IND Safety Reporting. Guidance for Industry. Draft Guidance December, 2015. Available from: http://www.fda.gov/ucm/ groups/fdagov-public/@fdagov-drugs gen/documents/document/ ucm477584.pdf. [Last accessed on 2016 Jan 06].

24. Michel DJ, Knodel LC. Program coordinated by a drug information service to improve adverse drug reaction reporting in a hospital. Am J Hosp Pharm 1986;43(9):2202-5.

25. Abideen PS. Practical implications of spontaneous adverse drug reaction reporting system in hospitals-an overview. Asian J Pharm Clin Res 2013;6(4):11. 